\title{
Controlled generalized fusion frame in the tensor product of Hilbert spaces
}

\author{
P. Ghosh and T. K. Samanta
}

\begin{abstract}
We present controlled by operators generalized fusion frame in the tensor product of Hilbert spaces and discuss some of its properties. We also describe the frame operator for a pair of controlled $g$-fusion Bessel sequences in the tensor product of Hilbert spaces.
\end{abstract}

Key Words: Frame, fusion frame, $g$-frame, $g$-fusion frame, frame operator, tensor product of Hilbert spaces, tensor product of frames Mathematics Subject Classification 2010: 42C15, 46C07, 46M05, 47A80

\section{Introduction}

Frame for a Hilbert space was first introduced by Duffin and Schaeffer [4] in 1952 to study some fundamental problems in non-harmonic Fourier series. In abstract Hilbert spaces, Daubechies et al. [3] gave the formal definition of frame in 1986. Frame theory has been widely used in signal and image processing, filter bank theory, coding and communications, etc. Several generalizations of frames, namely, $g$-frames [18], fusion frames [1], $g$-fusion frames [17], etc., have been introduced in recent times. A generalized fusion frame is used to generalize the theory of fusion frame and $g$-frame. Frame operator for the pair of $g$-fusion Bessel sequences was studied by the authors in [8], who also presented the stability of dual $g$-fusion frames in Hilbert spaces in [7].

The basic concept of the tensor product of Hilbert spaces was presented by Robinson [16]. Frames and bases in the tensor product of Hilbert spaces were introduced by Khosravi and Asgari [12]. Reddy et al. [19] studied the frame in the tensor product of Hilbert spaces and presented the tensor frame operator on the tensor product of Hilbert spaces. The concepts of fusion frames and $g$-frames in the tensor product of Hilbert spaces were introduced by Khosravi and Mirzaee Azandaryani [13]. Fusion frame and 
its alternative dual in the tensor product of Hilbert spaces were studied by the authors [9]. Also, we considered a generalized fusion frame in the tensor product of Hilbert spaces [10].

In this paper, we present a controlled generalized fusion frame in the tensor product of Hilbert spaces and establish some of its properties. The relation between the frame operators for the pair of controlled $g$-fusion Bessel sequences in Hilbert spaces and the tensor product of Hilbert spaces is also obtained.

Throughout this paper, $H$ and $K$ are considered to be separable Hilbert spaces with associated inner products $\langle\cdot, \cdot\rangle_{1}$ and $\langle\cdot, \cdot\rangle_{2}$, respectively. By $I_{H}$ and $I_{K}$ we denote the identity operators on $H$ and $K$, and $\mathcal{B}(H, K)$ is the collection of all bounded linear operators from $H$ to $K$. In particular, $\mathcal{B}(H)$ stands for the space of all bounded linear operators on $H$. By $P_{V}$ we denote the orthogonal projection onto the closed subspace $V \subset H$. Everywhere below, $I$ and $J$ stand for subsets of $\mathbb{Z}$. Let $\left\{V_{i}\right\}_{i \in I}$ and $\left\{W_{j}\right\}_{j \in J}$ be collections of closed subspaces of $H$ and $K,\left\{H_{i}\right\}_{i \in I}$ and $\left\{K_{j}\right\}_{j \in J}$ be collections of Hilbert spaces, $\left\{\Lambda_{i} \in \mathcal{B}\left(H, H_{i}\right)\right\}_{i \in I}$ and $\left\{\Gamma_{j} \in \mathcal{B}\left(K, K_{j}\right)\right\}_{j \in J}$ be sequences of operators. By $\mathcal{G B}(H)$ we denote the set of all bounded linear operators which have bounded inverse. If $S, R \in \mathcal{G B}(H)$, then $R^{*}, R^{-1}$ and $S R$ also belong to $\mathcal{G B}(H)$. Let $\mathcal{G B}^{+}(H)$ be the set of all positive operators in $\mathcal{G B}(H)$ and $T, U$ be invertible operators in $\mathcal{G B}(H)$.

For any $I \subset \mathbb{Z}$, define

$$
l^{2}\left(\left\{H_{i}\right\}_{i \in I}\right)=\left\{\left\{f_{i}\right\}_{i \in I}: f_{i} \in H_{i}, \sum_{i \in I}\left\|f_{i}\right\|^{2}<\infty\right\}
$$

with the inner product given by

$$
\left\langle\left\{f_{i}\right\}_{i \in I},\left\{g_{i}\right\}_{i \in I}\right\rangle=\sum_{i \in I}\left\langle f_{i}, g_{i}\right\rangle_{H_{i}} .
$$

Clearly (see also [17]), $l^{2}\left(\left\{H_{i}\right\}_{i \in I}\right)$ is a Hilbert space with respect to the above inner product. We can similarly define the space $l^{2}\left(\left\{K_{j}\right\}_{j \in J}\right)$.

\section{Preliminaries}

In this section, we recall some basic definitions and theorems.

Theorem 1 [6] Let $V \subset H$ be a closed subspace and $T \in \mathcal{B}(H)$. Then $P_{V} T^{*}=P_{V} T^{*} P_{\overline{T V}}$. If $T$ is a unitary operator, i.e., $T^{*} T=I_{H}$, then $P_{\overline{T V}} T=T P_{V}$.

Theorem 2 [2] The set $\mathcal{S}(H)$ of all self-adjoint operators on $H$ is a partially ordered set with respect to the partial order $\leq$ : for $T, S \in \mathcal{S}(H), T \leq S$ if and only if $\langle T f, f\rangle_{1} \leq\langle S f, f\rangle_{1}$ for all $f \in H$. 
A sequence $\left\{f_{i}\right\}_{i \in I}$ of elements in $H$ is called a frame for $H$ (see [2]) if there exist constants $A, B>0$ such that

$$
A\|f\|_{1}^{2} \leq \sum_{i \in I}\left|\left\langle f, f_{i}\right\rangle_{1}\right|^{2} \leq B\|f\|_{1}^{2}
$$

for all $f \in H$. The constants $A$ and $B$ are called frame bounds.

Let $\left\{v_{i}\right\}_{i \in I}$ be a collection of positive weights. The family $\Lambda=$ $\left\{\left(V_{i}, \Lambda_{i}, v_{i}\right)\right\}_{i \in I}$ is called a $g$-fusion frame for $H$ with respect to $\left\{H_{i}\right\}_{i \in I}$ if there exist constants $0<A \leq B<\infty$ such that

$$
A\|f\|_{1}^{2} \leq \sum_{i \in I} v_{i}^{2}\left\|\Lambda_{i} P_{V_{i}}(f)\right\|_{1}^{2} \leq B\|f\|_{1}^{2}, \quad f \in H .
$$

The constants $A$ and $B$ are called the lower and the upper bounds of $g$-fusion frame, respectively. If $A=B$, then $\Lambda$ is called tight $g$-fusion frame, and if $A=B=1$, we say that $\Lambda$ is a Parseval $g$-fusion frame. If $\Lambda$ satisfies only the right inequality in (1), it is called a $g$-fusion Bessel sequence with the bound $B$ in $H$ (see [17]).

Let $\Lambda=\left\{\left(V_{i}, \Lambda_{i}, v_{i}\right)\right\}_{i \in I}$ and $\Lambda^{\prime}=\left\{\left(V_{i}^{\prime}, \Lambda_{i}^{\prime}, v_{i}^{\prime}\right)\right\}_{i \in I}$ be two $g$-fusion Bessel sequences in $H$ with bounds $D_{1}$ and $D_{2}$, respectively. The operator $S_{\Lambda \Lambda^{\prime}}$ : $H \rightarrow H$ defined by

$$
S_{\Lambda \Lambda^{\prime}}(f)=\sum_{i \in I} v_{i} v_{i}^{\prime} P_{V_{i}} \Lambda_{i}^{*} \Lambda_{i}^{\prime} P_{V_{i}^{\prime}}(f), \quad f \in H,
$$

is called the frame operator for the pair of $g$-fusion Bessel sequences $\Lambda$ and $\Lambda^{\prime}$ (see [8]).

Now we recall the notion of a controlled $g$-fusion frame and related concepts (see [14]). Let $\left\{W_{j}\right\}_{j \in J}$ be a collection of closed subspaces of $H$ and $\left\{v_{j}\right\}_{j \in J}$ be a collection of positive weights. Let $\left\{H_{j}\right\}_{j \in J}$ be a sequence of Hilbert spaces, $T, U \in \mathcal{G B}(H)$ and $\Lambda_{j} \in \mathcal{B}\left(H, H_{j}\right), j \in J$. The family $\Lambda_{T U}=\left\{\left(W_{j}, \Lambda_{j}, v_{j}\right)\right\}_{j \in J}$ is called a $(T, U)$-controlled $g$-fusion frame for $H$ if there exist constants $0<A \leq B<\infty$ such that

$$
A\|f\|_{1}^{2} \leq \sum_{j \in J} v_{j}^{2}\left\langle\Lambda_{j} P_{W_{j}} U f, \Lambda_{j} P_{W_{j}} T f\right\rangle_{1} \leq B\|f\|_{1}^{2}, \quad f \in H .
$$

If $A=B$, then $\Lambda_{T U}$ is called $(T, U)$-controlled tight $g$-fusion frame and if $A=B=1$, we say that $\Lambda_{T U}$ is a $(T, U)$-controlled Parseval $g$-fusion frame. If $\Lambda_{T U}$ satisfies only the right inequality in (2), it is called a $(T, U)$-controlled $g$-fusion Bessel sequence in $H$.

Let $\Lambda_{T U}$ be a $(T, U)$-controlled $g$-fusion Bessel sequence in $H$ with a bound $B$. The synthesis operator $T_{C}: \mathcal{K}_{\Lambda_{j}} \rightarrow H$ is defined by

$$
T_{C}\left(\left\{v_{j}\left(T^{*} P_{W_{j}} \Lambda_{j}^{*} \Lambda_{j} P_{W_{j}} U\right)^{1 / 2} f\right\}_{j \in J}\right)=\sum_{j \in J} v_{j}^{2} T^{*} P_{W_{j}} \Lambda_{j}^{*} \Lambda_{j} P_{W_{j}} U f,
$$


and the analysis operator $T_{C}^{*}: H \rightarrow \mathcal{K}_{\Lambda_{j}}$ is given by

$$
T_{C}^{*} f=\left\{v_{j}\left(T^{*} P_{W_{j}} \Lambda_{j}^{*} \Lambda_{j} P_{W_{j}} U\right)^{1 / 2} f\right\}_{j \in J}, \quad f \in H
$$

where

$$
\mathcal{K}_{\Lambda_{j}}=\left\{\left\{v_{j}\left(T^{*} P_{W_{j}} \Lambda_{j}^{*} \Lambda_{j} P_{W_{j}} U\right)^{1 / 2} f\right\}_{j \in J}: f \in H\right\} \subset l^{2}\left(\left\{H_{j}\right\}_{j \in J}\right),
$$

$J \subset \mathbb{Z}$. The frame operator $S_{C}: H \rightarrow H$ is defined as follows

$$
S_{C} f=T_{C} T_{C}^{*} f=\sum_{j \in J} v_{j}^{2} T^{*} P_{W_{j}} \Lambda_{j}^{*} \Lambda_{j} P_{W_{j}} U f, \quad f \in H,
$$

and it is easy to verify that for any $f \in H$,

$$
\left\langle S_{C} f, f\right\rangle_{1}=\sum_{j \in J} v_{j}^{2}\left\langle\Lambda_{j} P_{W_{j}} U f, \Lambda_{j} P_{W_{j}} T f\right\rangle_{1} .
$$

Furthermore, if $\Lambda_{T U}$ is a $(T, U)$-controlled $g$-fusion frame with bounds $A$ and $B$, then $A I_{H} \leq S_{C} \leq B I_{H}$. Hence, $S_{C}$ is bounded, invertible, self-adjoint and positive linear operator. It is easy to verify that $B^{-1} I_{H} \leq S_{C}^{-1} \leq A^{-1} I_{H}$ (see [14]).

Let $K \in \mathcal{B}(H),\left\{W_{j}\right\}_{j \in J}$ be a collection of closed subspaces of $H$ and $\left\{v_{j}\right\}_{j \in J}$ be a collection of positive weights. Let $\left\{H_{j}\right\}_{j \in J}$ be a sequence of Hilbert spaces, $T, U \in \mathcal{G B}(H)$ and $\Lambda_{j} \in \mathcal{B}\left(H, H_{j}\right)$ for each $j \in J$. The family $\Lambda_{T U}=\left\{\left(W_{j}, \Lambda_{j}, v_{j}\right)\right\}_{j \in J}$ is called a $(T, U)$-controlled $K$-g-fusion frame for $H$ (see [15]) if there exist constants $0<A \leq B<\infty$ such that

$$
A\left\|K^{*} f\right\|_{1}^{2} \leq \sum_{j \in J} v_{j}^{2}\left\langle\Lambda_{j} P_{W_{j}} U f, \Lambda_{j} P_{W_{j}} T f\right\rangle_{1} \leq B\|f\|_{1}^{2}, \quad f \in H .
$$

There are several ways to introduce the tensor product of Hilbert spaces. The tensor product of Hilbert spaces $H$ and $K$ is a certain linear space of operators which was presented by Folland [5] and independently by Kadison and Ringrose [11]. Below we give the definition according to [19].

The tensor product of Hilbert spaces $H$ and $K$ is denoted by $H \otimes K$ and is defined to be an inner product space associated with the inner product

$$
\left\langle f \otimes g, f^{\prime} \otimes g^{\prime}\right\rangle=\left\langle f, f^{\prime}\right\rangle_{1}\left\langle g, g^{\prime}\right\rangle_{2}
$$

for all $f, f^{\prime} \in H$ and $g, g^{\prime} \in K$. The norm on $H \otimes K$ is given by

$$
\|f \otimes g\|=\|f\|_{1}\|g\|_{2}, \quad f \in H, g \in K .
$$

The space $H \otimes K$ is complete with respect to the above inner product. Therefore, the space $H \otimes K$ is a Hilbert space. 
For $Q \in \mathcal{B}(H)$ and $T \in \mathcal{B}(K)$, the tensor product of operators $Q$ and $T$ is denoted by $Q \otimes T$ and is defined as

$$
(Q \otimes T) A=Q A T^{*}, \quad A \in H \otimes K .
$$

It can be easily verified that $Q \otimes T \in \mathcal{B}(H \otimes K)$ (see, for example, [5]).

Theorem 3 [5] Suppose $Q, Q^{\prime} \in \mathcal{B}(H)$ and $T, T^{\prime} \in \mathcal{B}(K)$. Then

(i) $Q \otimes T \in \mathcal{B}(H \otimes K)$ and $\|Q \otimes T\|=\|Q\|\|T\|$;

(ii) $(Q \otimes T)(f \otimes g)=Q(f) \otimes T(g)$ for all $f \in H, g \in K$;

$($ iii $)(Q \otimes T)\left(Q^{\prime} \otimes T^{\prime}\right)=\left(Q Q^{\prime}\right) \otimes\left(T T^{\prime}\right)$;

(iv) $Q \otimes T$ is invertible if and only if $Q$ and $T$ are invertible, in which case $(Q \otimes T)^{-1}=\left(Q^{-1} \otimes T^{-1}\right) ;$

$(v)(Q \otimes T)^{*}=\left(Q^{*} \otimes T^{*}\right)$;

(vi) $I_{H} \otimes I_{K}=I_{H \otimes K}$, where $I_{H \otimes K}$ is the identity operator on $H \otimes K$.

\section{Controlled $g$-fusion frame in $H \otimes K$}

In this section, we present the notion of a controlled $g$-fusion frame in tensor product of Hilbert spaces. This concept is a generalization of the notion of $g$-fusion frame in $H \otimes K$.

Let $\left\{v_{i}\right\}_{i \in I}$ and $\left\{w_{j}\right\}_{j \in J}$ be two families of positive weights. Suppose $T, U \in \mathcal{G B}(H), T_{1}, U_{1} \in \mathcal{G} \mathcal{B}(K)$ and $\Lambda_{i} \in \mathcal{B}\left(H, H_{i}\right), \Gamma_{j} \in \mathcal{B}\left(K, K_{j}\right)$ for each $i \in I, j \in J$. The family $\Delta=\left\{\left(V_{i} \otimes W_{j}, \Lambda_{i} \otimes \Gamma_{j}, v_{i} w_{j}\right)\right\}_{i \in I, j \in J}$ is called a generalized fusion frame controlled by the operators $\left(T \otimes T_{1}, U \otimes U_{1}\right)$ (or $\left(T \otimes T_{1}, U \otimes U_{1}\right)$-controlled $g$-fusion frame) for $H \otimes K$ with respect to $\left\{H_{i} \otimes K_{j}\right\}_{i \in I, j \in J}$ if there exist constants $0<A \leq B<\infty$ such that

$$
\begin{aligned}
& A\|h\|^{2} \\
& \leq \sum_{i, j} v_{i}^{2} w_{j}^{2}\left\langle\Delta_{i j} P_{V_{i} \otimes W_{j}}\left(U \otimes U_{1}\right) h, \Delta_{i j} P_{V_{i} \otimes W_{j}}\left(T \otimes T_{1}\right) h\right\rangle \\
& \leq B\|h\|^{2}
\end{aligned}
$$

$h=f \otimes g \in H \otimes K$, where $P_{V_{i} \otimes W_{j}}$ is the orthogonal projection of $H \otimes K$ onto $V_{i} \otimes W_{j}$ and $\Delta_{i j}=\Lambda_{i} \otimes \Gamma_{j}$. The constants $A$ and $B$ are called the frame bounds. If $A=B$, then it is called a $\left(T \otimes T_{1}, U \otimes U_{1}\right)$-controlled tight $g$-fusion frame.

Let us make several remarks.

(i) If the family $\Delta$ satisfies only the right inequality in (5), it is called 
a $\left(T \otimes T_{1}, U \otimes U_{1}\right)$-controlled $g$-fusion Bessel sequence in $H \otimes K$ with the bound $B$.

(ii) If $T=I_{H}$ and $T_{1}=I_{K}$, then $\Delta$ is called a $\left(I_{H \otimes K}, U \otimes U_{1}\right)$-controlled $g$-fusion frame for $H \otimes K$.

(iii) If $T=U=I_{H}$ and $T_{1}=U_{1}=I_{K}$, then $\Delta$ is called a $\left(I_{H \otimes K}, I_{H \otimes K}\right)$ controlled $g$-fusion frame for $H \otimes K$. In this case, inequalities (5) can be written as follows

$$
A\|f \otimes g\|^{2} \leq \sum_{i, j} v_{i}^{2} w_{j}^{2}\left\|\left(\Lambda_{i} \otimes \Gamma_{j}\right) P_{V_{i} \otimes W_{j}}(f \otimes g)\right\|^{2} \leq B\|f \otimes g\|^{2},
$$

for all $f \otimes g \in H \otimes K$. Hence, $\Delta$ is a $g$-fusion frame for $H \otimes K$. For more details on $g$-fusion frame in the tensor product of Hilbert spaces, see [10].

For $i \in I$ and $j \in J$, define the space

$$
l^{2}\left(\left\{H_{i} \otimes K_{j}\right\}\right)=\left\{\left\{f_{i} \otimes g_{j}\right\}: f_{i} \otimes g_{j} \in H_{i} \otimes K_{j}, \sum_{i, j}\left\|f_{i} \otimes g_{j}\right\|^{2}<\infty\right\}
$$

with the inner product

$$
\begin{aligned}
& \left\langle\left\{f_{i} \otimes g_{j}\right\},\left\{f_{i}^{\prime} \otimes g_{j}^{\prime}\right\}\right\rangle_{l^{2}}=\sum_{i, j}\left\langle f_{i} \otimes g_{j}, f_{i}^{\prime} \otimes g_{j}^{\prime}\right\rangle \\
& =\sum_{i, j}\left\langle f_{i}, f_{i}^{\prime}\right\rangle_{H_{i}}\left\langle g_{j}, g_{j}^{\prime}\right\rangle_{K_{j}}=\left(\sum_{i \in I}\left\langle f_{i}, f_{i}^{\prime}\right\rangle_{H_{i}}\right)\left(\sum_{j \in J}\left\langle g_{j}, g_{j}^{\prime}\right\rangle_{K_{j}}\right) \\
& =\left\langle\left\{f_{i}\right\}_{i \in I},\left\{f_{i}^{\prime}\right\}_{i \in I}\right\rangle_{l^{2}\left(\left\{H_{i}\right\}_{i \in I}\right)}\left\langle\left\{g_{j}\right\}_{j \in J},\left\{g_{j}^{\prime}\right\}_{j \in J}\right\rangle_{l^{2}\left(\left\{K_{j}\right\}_{j \in J}\right)} .
\end{aligned}
$$

The space $l^{2}\left(\left\{H_{i} \otimes K_{j}\right\}\right)$ is complete with the above inner product. Therefore, it is a Hilbert space with respect to the above inner product.

Since $\left\{V_{i}\right\}_{i \in I},\left\{W_{j}\right\}_{j \in J}$ and $\left\{V_{i} \otimes W_{j}\right\}_{i, j}$ are the families of closed subspaces of the Hilbert spaces $H, K$ and $H \otimes K$, respectively, it is easy to verify that $P_{V_{i} \otimes W_{j}}=P_{V_{i}} \otimes P_{W_{j}}$.

For the remaining part of this paper, we denote $\Lambda_{i} \otimes \Gamma_{j}$ by $\Delta_{i j}$, and the families $\left\{\left(V_{i}, \Lambda_{i}, v_{i}\right)\right\}_{i \in I}$ and $\left\{\left(W_{j}, \Gamma_{j}, w_{j}\right)\right\}_{j \in J}$ by $\Lambda_{T U}$ and $\Gamma_{T_{1} U_{1}}$, respectively.

Theorem 4 The family $\Delta=\left\{\left(V_{i} \otimes W_{j}, \Lambda_{i} \otimes \Gamma_{j}, v_{i} w_{j}\right)\right\}_{i \in I, j \in J}$ is a $\left(T \otimes T_{1}, U \otimes U_{1}\right)$-controlled $g$-fusion frame for $H \otimes K$ with respect to $\left\{H_{i} \otimes K_{j}\right\}_{i \in I, j \in J}$ if and only if $\Lambda_{T U}$ is a $(T, U)$-controlled $g$-fusion frame for $H$ with respect to $\left\{H_{i}\right\}_{i \in I}$ and $\Gamma_{T_{1} U_{1}}$ is a $\left(T_{1}, U_{1}\right)$-controlled $g$-fusion frame for $K$ with respect to $\left\{K_{j}\right\}_{j \in J}$.

Proof. First, suppose that $\Delta$ is a $\left(T \otimes T_{1}, U \otimes U_{1}\right)$-controlled $g$-fusion frame for $H \otimes K$ with respect to $\left\{H_{i} \otimes K_{j}\right\}_{i \in I, j \in J}$ having bounds $A$ and $B$. Then 
for each $h=f \otimes g \in H \otimes K-\{\theta \otimes \theta\}$, using (3) and (4), we have

$$
A\|h\|^{2} \leq \sum_{i, j} v_{i}^{2} w_{j}^{2}\left\langle\Delta_{i j} P_{V_{i} \otimes W_{j}}\left(U \otimes U_{1}\right) h, \Delta_{i j} P_{V_{i} \otimes W_{j}}\left(T \otimes T_{1}\right) h\right\rangle \leq B\|h\|^{2},
$$

and hence,

$$
A\|h\|^{2} \leq \sum_{i, j} v_{i}^{2} w_{j}^{2}\left\langle\Lambda_{i} P_{V_{i}} U f \otimes \Gamma_{j} P_{W_{j}} U_{1} g, \Lambda_{i} P_{V_{i}} T f \otimes \Gamma_{j} P_{W_{j}} T_{1} g\right\rangle \leq B\|h\|^{2} .
$$

Further,

$$
\begin{gathered}
A\|f\|_{1}^{2}\|g\|_{2}^{2} \leq \sum_{i, j} v_{i}^{2} w_{j}^{2}\left\langle\Lambda_{i} P_{V_{i}} U f, \Lambda_{i} P_{V_{i}} T f\right\rangle_{1}\left\langle\Gamma_{j} P_{W_{j}} U_{1} g, \Gamma_{j} P_{W_{j}} T_{1} g\right\rangle_{2} \\
\leq B\|f\|_{1}^{2}\|g\|_{2}^{2}
\end{gathered}
$$

and thus,

$$
\begin{gathered}
A\|f\|_{1}^{2}\|g\|_{2}^{2} \leq \sum_{i \in I} v_{i}^{2}\left\langle\Lambda_{i} P_{V_{i}} U f, \Lambda_{i} P_{V_{i}} T f\right\rangle_{1} \sum_{j \in J} w_{j}^{2}\left\langle\Gamma_{j} P_{W_{j}} U_{1} g, \Gamma_{j} P_{W_{j}} T_{1} g\right\rangle_{2} \\
\leq B\|f\|_{1}^{2}\|g\|_{2}^{2} .
\end{gathered}
$$

Since $f \otimes g$ is non-zero vector, $f$ and $g$ are also non-zero vectors, and therefore,

$$
\sum_{i \in I} v_{i}^{2}\left\langle\Lambda_{i} P_{V_{i}} U f, \Lambda_{i} P_{V_{i}} T f\right\rangle_{1}, \quad \sum_{j \in J} w_{j}^{2}\left\langle\Gamma_{j} P_{W_{j}} U_{1} g, \Gamma_{j} P_{W_{j}} T_{1} g\right\rangle_{2}
$$

are non-zero. Thus, from the above inequality, we get

$$
\begin{gathered}
\frac{A\|g\|_{2}^{2}\|f\|_{1}^{2}}{\sum_{j \in J} w_{j}^{2}\left\langle\Gamma_{j} P_{W_{j}} U_{1} g, \Gamma_{j} P_{W_{j}} T_{1} g\right\rangle_{2}} \leq \sum_{i \in I} v_{i}^{2}\left\langle\Lambda_{i} P_{V_{i}} U f, \Lambda_{i} P_{V_{i}} T f\right\rangle_{1} \\
\leq \frac{B\|g\|_{2}^{2}\|f\|_{1}^{2}}{\sum_{j \in J} w_{j}^{2}\left\langle\Gamma_{j} P_{W_{j}} U_{1} g, \Gamma_{j} P_{W_{j}} T_{1} g\right\rangle_{2}} .
\end{gathered}
$$

Then

$$
A_{1}\|f\|_{1}^{2} \leq \sum_{i \in I} v_{i}^{2}\left\langle\Lambda_{i} P_{V_{i}} U f, \Lambda_{i} P_{V_{i}} T f\right\rangle_{1} \leq B_{1}\|f\|_{1}^{2}, \quad f \in H
$$

where

$$
A_{1}=\inf _{g \in K} \frac{A\|g\|_{2}^{2}}{\sum_{j \in J} w_{j}^{2}\left\langle\Gamma_{j} P_{W_{j}} U_{1} g, \Gamma_{j} P_{W_{j}} T_{1} g\right\rangle_{2}}
$$


and

$$
B_{1}=\sup _{g \in K} \frac{B\|g\|_{2}^{2}}{\sum_{j \in J} w_{j}^{2}\left\langle\Gamma_{j} P_{W_{j}} U_{1} g, \Gamma_{j} P_{W_{j}} T_{1} g\right\rangle_{2}} .
$$

This shows that $\Lambda_{T U}$ is a $(T, U)$-controlled $g$-fusion frame for $H$ with respect to $\left\{H_{i}\right\}_{i \in I}$. Similarly, it can be shown that $\Gamma_{T_{1} U_{1}}$ is $\left(T_{1}, U_{1}\right)$-controlled $g$ fusion frame for $K$ with respect to $\left\{K_{j}\right\}_{j \in J}$.

Conversely, suppose that $\Lambda_{T U}$ is a $(T, U)$-controlled $g$-fusion frame for $H$ with respect to $\left\{H_{i}\right\}_{i \in I}$ and $\Gamma_{T_{1} U_{1}}$ is a $\left(T_{1}, U_{1}\right)$-controlled $g$-fusion frame for $K$ with respect to $\left\{K_{j}\right\}_{j \in J}$ having bounds $A, B$ and $C, D$, respectively. Then

$$
\begin{gathered}
A\|f\|_{1}^{2} \leq \sum_{i \in I} v_{i}^{2}\left\langle\Lambda_{i} P_{V_{i}} U f, \Lambda_{i} P_{V_{i}} T f\right\rangle_{1} \leq B\|f\|_{1}^{2}, \quad f \in H, \\
C\|g\|_{2}^{2} \leq \sum_{j \in J} w_{j}^{2}\left\langle\Gamma_{j} P_{W_{j}} U_{1} g, \Gamma_{j} P_{W_{j}} T_{1} g\right\rangle_{2} \leq D\|g\|_{2}^{2}, \quad g \in K .
\end{gathered}
$$

Multiplying (6) with (7) and using (3) and (4), we get

$$
\begin{gathered}
A C\|f \otimes g\|^{2} \leq \sum_{i, j} v_{i}^{2} w_{j}^{2}\left\langle\Lambda_{i} P_{V_{i}} U f, \Lambda_{i} P_{V_{i}} T f\right\rangle_{1}\left\langle\Gamma_{j} P_{W_{j}} U_{1} g, \Gamma_{j} P_{W_{j}} T_{1} g\right\rangle_{2} \\
\leq B D\|f \otimes g\|^{2}, \\
A\|f \otimes g\|^{2} \leq \sum_{i, j} v_{i}^{2} w_{j}^{2}\left\langle\Lambda_{i} P_{V_{i}} U f \otimes \Gamma_{j} P_{W_{j}} U_{1} g, \Lambda_{i} P_{V_{i}} T f \otimes \Gamma_{j} P_{W_{j}} T_{1} g\right\rangle \\
\leq B\|f \otimes g\|^{2}, \\
A C\|h\|^{2} \leq \sum_{i, j} v_{i}^{2} w_{j}^{2}\left\langle\Delta_{i j} P_{V_{i} \otimes W_{j}}\left(U \otimes U_{1}\right) h, \Delta_{i j} P_{V_{i} \otimes W_{j}}\left(T \otimes T_{1}\right) h\right\rangle \\
\leq B D\|h\|^{2}
\end{gathered}
$$

for all $h=f \otimes g \in H \otimes K$. Hence, $\Delta$ is a $\left(T \otimes T_{1}, U \otimes U_{1}\right)$-controlled $g$ fusion frame for $H \otimes K$ with respect to $\left\{H_{i} \otimes K_{j}\right\}_{i \in I, j \in J}$ with bounds $A C$ and $B D$. This completes the proof.

Note, that Theorem 3.4 in [10] can be obtained as a corollary of Theorem 4 by substituting $T=U=I_{H}$ and $T_{1}=U_{1}=I_{K}$.

Now, we validate this theorem by considering the following example.

Example. Let $H=\mathbb{R}^{3}$ and $\left\{e_{1}, e_{2}, e_{3}\right\}$ be an orthonormal basis for $H$. Suppose that $V_{1}=\overline{\operatorname{span}}\left\{e_{2}, e_{3}\right\}, V_{2}=\overline{\operatorname{span}}\left\{e_{1}, e_{3}\right\}$ and $V_{3}=\overline{\operatorname{span}}\left\{e_{1}, e_{2}\right\}$ 
with $V_{i}=H_{i}, i=1,2,3$. Define $\Lambda_{1} f=\left\langle f, e_{2}\right\rangle e_{3}, \Lambda_{2} f=\left\langle f, e_{3}\right\rangle e_{1}$ and $\Lambda_{3} f=\left\langle f, e_{1}\right\rangle e_{2}$. Consider the following two operators on $H$ :

$$
T\left(f_{1}, f_{2}, f_{3}\right)=\left(2 f_{1}, 3 f_{2}, 5 f_{3}\right), \quad U\left(f_{1}, f_{2}, f_{3}\right)=\left(\frac{f_{1}}{2}, \frac{f_{2}}{3}, \frac{f_{3}}{4}\right) .
$$

It is easy to verify that $T, U \in \mathcal{G B}^{+}(H), T U=U T$. Now, for any $f=$ $\left(f_{1}, f_{2}, f_{3}\right) \in H$,

$$
\sum_{i=1}^{3}\left\langle\Lambda_{i} P_{V_{i}} U f, \Lambda_{i} P_{V_{i}} T f\right\rangle=f_{1}^{2}+f_{2}^{2}+\frac{5}{4} f_{3}^{2} .
$$

Thus,

$$
\|f\|^{2} \leq \sum_{i=1}^{3}\left\langle\Lambda_{i} P_{V_{i}} U f, \Lambda_{i} P_{V_{i}} T f\right\rangle \leq \frac{5}{4}\|f\|^{2}, \quad f \in H .
$$

Hence, $\left\{\left(V_{i}, \Lambda_{i}, 1\right)\right\}_{i=1}^{3}$ is a $(T, U)$-controlled $g$-fusion frame for $H$ with bounds 1 and $5 / 4$.

Next, we consider the Hilbert space $K=\mathbb{R}^{2}$ with an orthonormal basis $\left\{e_{1}, e_{2}\right\}$. Let $W_{j}=\overline{\operatorname{span}}\left\{e_{j}\right\}, j=1,2$, with $W_{j}=K_{j}, j=1,2$. Define $\Gamma_{1} g=\left\langle g, e_{1}\right\rangle e_{1}, \Gamma_{2} g=\left\langle g, e_{2}\right\rangle e_{2}$. Consider two operators on $H$ defined by

$$
T_{1}\left(g_{1}, g_{2}\right)=\left(5 g_{1}, 4 g_{2}\right), \quad U_{1}\left(g_{1}, g_{2}\right)=\left(\frac{g_{1}}{6}, \frac{g_{2}}{3}\right) .
$$

It is easy to verify that $T, U \in \mathcal{G B}^{+}(H)$ and $T U=U T$. Then, for any $g=\left(g_{1}, g_{2}\right) \in H$, we have

$$
\sum_{j=1}^{2}\left\langle\Gamma_{j} P_{W_{j}} U_{1} g, \Gamma_{j} P_{W_{j}} T_{1} g\right\rangle=\frac{5}{6} g_{1}^{2}+\frac{4}{3} g_{2}^{2} .
$$

Thus, $\left\{\left(W_{j}, \Gamma_{j}, 1\right)\right\}_{j=1}^{2}$ is a $\left(T_{1}, U_{1}\right)$-controlled $g$-fusion frame for $K$ with bounds $5 / 6$ and 4/3. Therefore, according to Theorem 4, the family $\left\{\left(V_{i} \otimes W_{j}, \Lambda_{i} \otimes \Gamma_{j}, 1\right)\right\}_{i \in I, j \in J}$ is a $\left(T \otimes T_{1}, U \otimes U_{1}\right)$-controlled $g$-fusion frame for $H \otimes K=\mathbb{R}^{6}$ with bounds $5 / 6$ and $5 / 3$.

Remark 1 Let $\Delta$ be a $\left(T \otimes T_{1}, U \otimes U_{1}\right)$-controlled g-fusion frame for the Hilbert space $H \otimes K$. According to its definition, the frame operator $S_{\Delta}$ : $H \otimes K \rightarrow H \otimes K$ is described by

$$
S_{\Delta} h=\sum_{i, j} v_{i}^{2} w_{j}^{2}\left(U \otimes U_{1}\right)^{*} P_{V_{i} \otimes W_{j}}\left(\Lambda_{i} \otimes \Gamma_{j}\right)^{*}\left(\Lambda_{i} \otimes \Gamma_{j}\right) P_{V_{i} \otimes W_{j}}\left(T \otimes T_{1}\right) h
$$

for all $h=f \otimes g \in H \otimes K$. 
Theorem 5 If $S_{T U}, S_{T_{1} U_{1}}$ and $S_{\Delta}$ are the corresponding frame operators for the controlled $g$-fusion frames $\Lambda_{T U}, \Gamma_{T_{1} U_{1}}$ and $\Delta$, respectively, then $S_{\Delta}=$ $S_{T U} \otimes S_{T_{1} U_{1}}$ and $S_{\Delta}^{-1}=S_{T U}^{-1} \otimes S_{T_{1} U_{1}}^{-1}$.

Proof. For each $h=f \otimes g \in H \otimes K$, we have

$$
\begin{aligned}
& S_{\Delta} h=\sum_{i, j} v_{i}^{2} w_{j}^{2}\left(U \otimes U_{1}\right)^{*} P_{V_{i} \otimes W_{j}}\left(\Lambda_{i} \otimes \Gamma_{j}\right)^{*}\left(\Lambda_{i} \otimes \Gamma_{j}\right) P_{V_{i} \otimes W_{j}}\left(T \otimes T_{1}\right) h \\
& =\sum_{i, j} v_{i}^{2} w_{j}^{2}\left(U^{*} \otimes U_{1}^{*}\right)\left(P_{V_{i}} \otimes P_{W_{j}}\right)\left(\Lambda_{i}^{*} \otimes \Gamma_{j}^{*}\right)\left(\Lambda_{i} \otimes \Gamma_{j}\right)\left(P_{V_{i}} T f \otimes P_{W_{j}} T_{1} g\right) \\
& =\sum_{i, j} v_{i}^{2} w_{j}^{2}\left(U^{*} P_{V_{i}} \Lambda_{i}^{*} \Lambda_{i} P_{V_{i}} T f \otimes U_{1}^{*} P_{W_{j}} \Gamma_{j}^{*} \Gamma_{j} P_{W_{j}} T_{1} g\right) \\
& =\left(\sum_{i \in I} v_{i}^{2} U^{*} P_{V_{i}} \Lambda_{i}^{*} \Lambda_{i} P_{V_{i}} T f\right) \otimes\left(\sum_{j \in J} w_{j}^{2} U_{1}^{*} P_{W_{j}} \Gamma_{j}^{*} \Gamma_{j} P_{W_{j}} T_{1} g\right) \\
& =S_{T U} f \otimes S_{T_{1} U_{1}} g=\left(S_{T U} \otimes S_{T_{1} U_{1}}\right)(f \otimes g) .
\end{aligned}
$$

This shows that $S_{\Delta}=S_{T U} \otimes S_{T_{1} U_{1}}$. Since $S_{T U}$ and $S_{T_{1} U_{1}}$ are invertible, by (iv) of Theorem 3 , it follows that

$$
S_{\Delta}^{-1}=\left(S_{T U} \otimes S_{T_{1} U_{1}}\right)^{-1}=S_{T U}^{-1} \otimes S_{T_{1} U_{1}}^{-1} .
$$

This completes the proof.

Theorem 6 If $S_{T U}, S_{T_{1} U_{1}}$ and $S_{\Delta}$ are the corresponding frame operators for the controlled $g$-fusion frames $\Lambda_{T U}, \Gamma_{T_{1} U_{1}}$ and $\Delta$, respectively. Then

$$
A C I_{H \otimes K} \leq S_{\Delta} \leq B D I_{H \otimes K},
$$

where $(A, B)$ and $(C, D)$ are frame bounds of $\Lambda_{T U}$ and $\Gamma_{T_{1} U_{1}}$, respectively, and $I_{H \otimes K}$ is the identity operator on $H \otimes K$.

Proof. Suppose $\Lambda_{T U}$ is a $(T, U)$-controlled $g$-fusion frame for $H$ with respect to $\left\{H_{i}\right\}_{i \in I}$ and $\Gamma_{T_{1} U_{1}}$ is a $\left(T_{1}, U_{1}\right)$-controlled $g$-fusion frame for $K$ with respect to $\left\{K_{j}\right\}_{j \in J}$ having bounds $A, B$ and $C, D$, respectively. Then

$$
A I_{H} \leq S_{T U} \leq B I_{H}, \quad C I_{K} \leq S_{T_{1} U_{1}} \leq D I_{K} .
$$

Taking tensor product of the above two inequalities, we get

$$
A C\left(I_{H} \otimes I_{K}\right) \leq S_{T U} \otimes S_{T_{1} U_{1}} \leq B D\left(I_{H} \otimes I_{K}\right) .
$$

Hence,

$$
A C I_{H \otimes K} \leq S_{\Delta} \leq B D I_{H \otimes K}
$$


The frame decomposition formula is the most important result in the frame theory. It shows that if $\Lambda_{T U}$ and $\Gamma_{T_{1} U_{1}}$ are controlled $g$-fusion frames in $H$ and $K$, respectively, then every element in $H \otimes K$ has a representation as a superposition of the frame elements. Now, we present the frame decomposition formula in $H \otimes K$.

Theorem 7 Let $\Lambda_{T U}$ be a $(T, U)$-controlled $g$-fusion frame for $H$ and $\Gamma_{T_{1} U_{1}}$ be a $\left(T_{1}, U_{1}\right)$-controlled $g$-fusion frames for $K$ with the corresponding frame operators $S_{T U}$ and $S_{T_{1} U_{1}}$, respectively. Then for each $f \otimes g \in H \otimes K$, we have

$$
f \otimes g=\sum_{i, j} v_{i}^{2} w_{j}^{2} S_{\Delta}^{-1}\left(U \otimes U_{1}\right)^{*} P_{V_{i} \otimes W_{j}}\left(\Lambda_{i} \otimes \Gamma_{j}\right)^{*}\left(\Lambda_{i} \otimes \Gamma_{j}\right) P_{V_{i} \otimes W_{j}} h
$$

and

$$
f \otimes g=\sum_{i, j} v_{i}^{2} w_{j}^{2}\left(U \otimes U_{1}\right)^{*} P_{V_{i} \otimes W_{j}}\left(\Lambda_{i} \otimes \Gamma_{j}\right)^{*}\left(\Lambda_{i} \otimes \Gamma_{j}\right) P_{V_{i} \otimes W_{j}} S_{\Delta}^{-1} h,
$$

where $h=\left(T \otimes T_{1}\right)(f \otimes g)$.

Proof. Since $S_{T U}$ and $S_{T_{1} U_{1}}$ are the corresponding frame operators for $\Lambda_{T U}$ and $\Gamma_{T_{1} U_{1}}$, respectively, for each $f \in H$ and $g \in K$, we have

$$
\begin{aligned}
& f=S_{T U} S_{T U}^{-1} f=\sum_{i \in I} v_{i}^{2} U^{*} P_{V_{i}} \Lambda_{i}^{*} \Lambda_{i} P_{V_{i}} T S_{T U}^{-1} f, \\
& g=\sum_{j \in J} w_{j}^{2} U_{1}^{*} P_{W_{j}} \Gamma_{j}^{*} \Gamma_{j} P_{W_{j}} T_{1} S_{T_{1} U_{1}}^{-1} g .
\end{aligned}
$$

Then for each $f \otimes g \in H \otimes K$, we can write

$$
\begin{aligned}
f \otimes g= & \left(\sum_{i \in I} v_{i}^{2} U^{*} P_{V_{i}} \Lambda_{i}^{*} \Lambda_{i} P_{V_{i}} T S_{T U}^{-1} f\right) \otimes \\
& \left(\sum_{j \in J} w_{j}^{2} U_{1}^{*} P_{W_{j}} \Gamma_{j}^{*} \Gamma_{j} P_{W_{j}} T_{1} S_{T_{1} T_{1}}^{-1} g\right) \\
= & \sum_{i, j} v_{i}^{2} w_{j}^{2}\left(U^{*} P_{V_{i}} \Lambda_{i}^{*} \Lambda_{i} P_{V_{i}} T S_{T U}^{-1} f \otimes U_{1}^{*} P_{W_{j}} \Gamma_{j}^{*} \Gamma_{j} P_{W_{j}} T_{1} S_{T_{1} U_{1}}^{-1} g\right) \\
= & \sum_{i, j} v_{i}^{2} w_{j}^{2}\left(U \otimes U_{1}\right)^{*} P_{V_{i} \otimes W_{j}}\left(\Lambda_{i} \otimes \Gamma_{j}\right)^{*}\left(\Lambda_{i} \otimes \Gamma_{j}\right) P_{V_{i} \otimes W_{j}} S_{\Delta}^{-1} h,
\end{aligned}
$$

where $h=\left(T \otimes T_{1}\right)(f \otimes g)$. On the other hand, for each $f \otimes g \in H \otimes K$,

$$
\begin{aligned}
f \otimes g & =S_{T U}^{-1} S_{T U} f \otimes S_{T_{1} U_{1}}^{-1} S_{T_{1} U_{1}}^{-1} g \\
& =\sum_{i, j} v_{i}^{2} w_{j}^{2} S_{\Delta}^{-1}\left(U \otimes U_{1}\right)^{*} P_{V_{i} \otimes W_{j}}\left(\Lambda_{i} \otimes \Gamma_{j}\right)^{*}\left(\Lambda_{i} \otimes \Gamma_{j}\right) P_{V_{i} \otimes W_{j}} h .
\end{aligned}
$$

This completes the proof. 
To get the frame decomposition in $H \otimes K$, we need to find the operator $S_{\Delta}^{-1}$. However, from the practical point of view, this is difficult. To avoid such a problem, we consider controlled tight $g$-fusion frame.

Corollary 1 Let $\Lambda_{T U}$ be a $(T, U)$-controlled tight g-fusion frame for $H$ and let $\Gamma_{T_{1} U_{1}}$ be a $\left(T_{1}, U_{1}\right)$-controlled tight $g$-fusion frame for $K$ with bounds $A_{1}$ and $A_{2}$, respectively. Then for each $f \otimes g \in H \otimes K$,

$$
f \otimes g=\frac{1}{A_{1} A_{2}} \sum_{i, j} v_{i}^{2} w_{j}^{2}\left(U \otimes U_{1}\right)^{*} P_{V_{i} \otimes W_{j}}\left(\Lambda_{i} \otimes \Gamma_{j}\right)^{*}\left(\Lambda_{i} \otimes \Gamma_{j}\right) P_{V_{i} \otimes W_{j}} h,
$$

where $h=\left(T \otimes T_{1}\right)(f \otimes g)$.

In the next theorem, using controlled $K$-g-fusion frames and some elements of $\mathcal{B}(H)$ and $\mathcal{B}(K)$, we construct a new controlled $g$-fusion frame in $H \otimes K$.

Theorem 8 Let $K_{1} \in \mathcal{B}(H)$ and $K_{2} \in \mathcal{B}(K)$. Let $\Lambda_{T U}$ be $(T, U)$-controlled $K_{1}$-g-fusion frame for $H$ and let $\Gamma_{T_{1} U_{1}}$ be $\left(T_{1}, U_{1}\right)$-controlled $K_{2}$-g-fusion frame for $K$ with bounds $A, B$ and $C, D$ and frame operators $S_{T U}$ and $S_{T_{1} U_{1}}$, respectively. Let $C_{1} \in \mathcal{B}(H)$ and $C_{2} \in \mathcal{B}(K)$ be invertible operators such that $C_{1}^{*}$ commutes with $T$ and $U$, while $C_{2}^{*}$ commutes with $T_{1}$ and $U_{1}$, respectively. Then the family

$$
\Theta=\left\{\left(C_{1} \otimes C_{2}\right)\left(V_{i} \otimes W_{j}\right),\left(\Lambda_{i} \otimes \Gamma_{j}\right) P_{V_{i} \otimes W_{j}}\left(C_{1} \otimes C_{2}\right)^{*}, v_{i} w_{j}\right\}_{i \in I, j \in J}
$$

is a $\left(T \otimes T_{1}, U \otimes U_{1}\right)$-controlled $\left(C_{1} \otimes C_{2}\right)\left(K_{1} \otimes K_{2}\right)\left(C_{1} \otimes C_{2}\right)^{*}$-fusion frame for $H \otimes K$. The corresponding frame operator for $\Theta$ is

$$
\left(C_{1} \otimes C_{2}\right) S_{\Delta}\left(C_{1} \otimes C_{2}\right)^{*} .
$$

Proof. Take $\Theta_{i j}=\left(\Lambda_{i} \otimes \Gamma_{j}\right) P_{V_{i} \otimes W_{j}}\left(C_{1} \otimes C_{2}\right)^{*} P_{\left(C_{1} \otimes C_{2}\right)\left(V_{i} \otimes W_{j}\right)}$. Then by Theorems 1 and 3 , we get

$$
\begin{aligned}
\Theta_{i j} & =\left(\Lambda_{i} \otimes \Gamma_{j}\right)\left(P_{V_{i}} \otimes P_{W_{j}}\right)\left(C_{1}^{*} \otimes C_{2}^{*}\right)\left(P_{C_{1} V_{i}} \otimes P_{C_{2} W_{j}}\right) \\
& =\Lambda_{i} P_{V_{i}} C_{1}^{*} P_{C_{1} V_{i}} \otimes \Gamma_{j} P_{W_{j}} C_{2}^{*} P_{C_{2} W_{j}}=\Lambda_{i} P_{V_{i}} C_{1}^{*} \otimes \Gamma_{j} P_{W_{j}} C_{2}^{*} .
\end{aligned}
$$

Using the obtained relation, for each $f \otimes g \in H \otimes K$, we can write

$$
\begin{aligned}
& \sum_{i, j} v_{i}^{2} w_{j}^{2}\left\langle\Theta_{i j}\left(U \otimes U_{1}\right)(f \otimes g), \Theta_{i j}\left(T \otimes T_{1}\right)(f \otimes g)\right\rangle \\
& =\sum_{i, j} v_{i}^{2} w_{j}^{2}\left\langle\Lambda_{i} P_{V_{i}} C_{1}^{*} U f \otimes \Gamma_{j} P_{W_{j}} C_{2}^{*} U_{1} g, \Lambda_{i} P_{V_{i}} C_{1}^{*} T f \otimes \Gamma_{j} P_{W_{j}} C_{2}^{*} T_{1} g\right\rangle \\
& =\sum_{i, j} v_{i}^{2} w_{j}^{2}\left\langle\Lambda_{i} P_{V_{i}} C_{1}^{*} U f, \Lambda_{i} P_{V_{i}} C_{1}^{*} T f\right\rangle_{1}\left\langle\Gamma_{j} P_{W_{j}} C_{2}^{*} U_{1} g, \Gamma_{j} P_{W_{j}} C_{2}^{*} T_{1} g\right\rangle_{2}
\end{aligned}
$$




$$
\begin{aligned}
&= \sum_{i \in I} v_{i}^{2}\left\langle\Lambda_{i} P_{V_{i}} U C_{1}^{*} f, \Lambda_{i} P_{V_{i}} T C_{1}^{*} f\right\rangle_{1} \times \\
& \sum_{j \in J} w_{j}^{2}\left\langle\Gamma_{j} P_{W_{j}} U_{1} C_{2}^{*} g, \Gamma_{j} P_{W_{j}} T_{1} C_{2}^{*} g\right\rangle_{2} \\
& \leq B\left\|C_{1}^{*} f\right\|_{1}^{2} D\left\|C_{2}^{*} g\right\|_{2}^{2} \leq B D\left\|C_{1}\right\|^{2}\|f\|_{1}^{2}\left\|C_{2}\right\|^{2}\|g\|_{2} \\
&=B D\left\|C_{1} \otimes C_{2}\right\|^{2}\|f \otimes g\|^{2} .
\end{aligned}
$$

On the other hand, for each $f \otimes g \in H \otimes K$, we have

$$
\begin{aligned}
& \frac{A C}{\left\|C_{1} \otimes C_{2}\right\|^{2}}\left\|\left[\left(C_{1} \otimes C_{2}\right)\left(K_{1} \otimes K_{2}\right)\left(C_{1} \otimes C_{2}\right)^{*}\right]^{*}(f \otimes g)\right\|^{2} \\
& =\frac{A C}{\left\|C_{1} \otimes C_{2}\right\|^{2}}\left\|\left(C_{1} \otimes C_{2}\right)\left(K_{1}^{*} \otimes K_{2}^{*}\right)\left(C_{1}^{*} \otimes C_{2}^{*}\right)(f \otimes g)\right\|^{2} \\
& =\frac{A C}{\left\|C_{1} \otimes C_{2}\right\|^{2}}\left\|\left(C_{1} K_{1}^{*} C_{1}^{*} \otimes C_{2} K_{2}^{*} C_{2}^{*}\right)(f \otimes g)\right\|^{2} \\
& =\frac{A C}{\left\|C_{1}\right\|^{2}\left\|C_{2}\right\|^{2}}\left\|C_{1} K_{1}^{*} C_{1}^{*} f\right\|_{1}^{2}\left\|C_{2} K_{2}^{*} C_{2}^{*} g\right\|_{2}^{2} \\
& \leq A\left\|K_{1}^{*} C_{1}^{*} f\right\|_{1}^{2} C\left\|K_{2}^{*} C_{2}^{*} g\right\|_{2}^{2} \\
& \leq \sum_{i \in I} v_{i}^{2}\left\langle\Lambda_{i} P_{V_{i}} U C_{1}^{*} f, \Lambda_{i} P_{V_{i}} T C_{1}^{*} f\right\rangle_{1} \times \\
& \quad \sum_{j \in J} w_{j}^{2}\left\langle\Gamma_{j} P_{W_{j}} U_{1} C_{2}^{*} g, \Gamma_{j} P_{W_{j}} T_{1} C_{2}^{*} g\right\rangle_{2} \\
& =\sum_{i, j} v_{i}^{2} w_{j}^{2}\left\langle\Theta_{i j}\left(U \otimes U_{1}\right)(f \otimes g), \Theta_{i j}\left(T \otimes T_{1}\right)(f \otimes g)\right\rangle .
\end{aligned}
$$

Hence, $\Theta$ is a $\left(T \otimes T_{1}, U \otimes U_{1}\right)$-controlled $\left(C_{1} \otimes C_{2}\right)\left(K_{1} \otimes K_{2}\right)\left(C_{1} \otimes C_{2}\right)^{*}$ fusion frame for $H \otimes K$.

Further, take $\Delta=\left(\Lambda_{i} \otimes \Gamma_{j}\right) P_{V_{i} \otimes W_{j}}\left(C_{1} \otimes C_{2}\right)^{*}$. Then, applying Theorem 3, we obtain

$$
\begin{aligned}
\Delta^{*} \Delta & =\left(\left(\Lambda_{i} \otimes \Gamma_{j}\right) P_{V_{i} \otimes W_{j}}\left(C_{1} \otimes C_{2}\right)^{*}\right)^{*}\left(\Lambda_{i} \otimes \Gamma_{j}\right) P_{V_{i} \otimes W_{j}}\left(C_{1} \otimes C_{2}\right)^{*} \\
& =\left(C_{1} \otimes C_{2}\right)\left(P_{V_{i}} \otimes P_{W_{j}}\right)\left(\Lambda_{i}^{*} \otimes \Gamma_{j}^{*}\right)\left(\Lambda_{i} \otimes \Gamma_{j}\right)\left(P_{V_{i}} \otimes P_{W_{j}}\right)\left(C_{1}^{*} \otimes C_{2}^{*}\right) \\
& =C_{1} P_{V_{i}} \Lambda_{i}^{*} \Lambda_{i} P_{V_{i}} C_{1}^{*} \otimes C_{2} P_{W_{j}} \Gamma_{j}^{*} \Gamma_{j} P_{W_{j}} C_{2}^{*} .
\end{aligned}
$$

Now,

$$
\begin{aligned}
& P_{\left(C_{1} \otimes C_{2}\right)\left(V_{i} \otimes W_{j}\right)} \Delta^{*} \Delta P_{\left(C_{1} \otimes C_{2}\right)\left(V_{i} \otimes W_{j}\right)} \\
& =\left(P_{C_{1} V_{i}} \otimes P_{C_{2} W_{j}}\right) \Delta^{*} \Delta\left(P_{C_{1} V_{i}} \otimes P_{C_{2} W_{j}}\right) \\
& =P_{C_{1} V_{i}} C_{1} P_{V_{i}} \Lambda_{i}^{*} \Lambda_{i} P_{V_{i}} C_{1}^{*} P_{C_{1} V_{i}} \otimes P_{C_{2} W_{j}} C_{2} P_{W_{j}} \Gamma_{j}^{*} \Gamma_{j} P_{W_{j}} C_{2}^{*} P_{C_{2} W_{j}} \\
& =\left(P_{V_{i}} C_{1}^{*}\right)^{*} \Lambda_{i}^{*} \Lambda_{i} P_{V_{i}} C_{1}^{*} \otimes\left(P_{W_{j}} C_{2}^{*}\right)^{*} \Gamma_{j}^{*} \Gamma_{j} P_{W_{j}} C_{1}^{*} \\
& =C_{1} P_{V_{i}} \Lambda_{i}^{*} \Lambda_{i} P_{V_{i}} C_{1}^{*} \otimes C_{2} P_{W_{j}} \Gamma_{j}^{*} \Gamma_{j} P_{W_{j}} C_{2}^{*} .
\end{aligned}
$$


Therefore, for each $h=f \otimes g \in H \otimes K$, we have

$$
\begin{aligned}
& \sum_{i, j} v_{i}^{2} w_{j}^{2}\left(U \otimes U_{1}\right)^{*} P_{\left(C_{1} \otimes C_{2}\right)\left(V_{i} \otimes W_{j}\right)} \Delta^{*} \Delta P_{\left(C_{1} \otimes C_{2}\right)\left(V_{i} \otimes W_{j}\right)}\left(T \otimes T_{1}\right) h \\
& =\sum_{i, j} v_{i}^{2} w_{j}^{2}\left(U^{*} \otimes U_{1}^{*}\right)\left(C_{1} P_{V_{i}} \Lambda_{i}^{*} \Lambda_{i} P_{V_{i}} C_{1}^{*} \otimes C_{2} P_{W_{j}} \Gamma_{j}^{*} \Gamma_{j} P_{W_{j}} C_{2}^{*}\right)\left(T f \otimes T_{1} g\right) \\
& =\sum_{i, j} v_{i}^{2} w_{j}^{2}\left(U^{*} C_{1} P_{V_{i}} \Lambda_{i}^{*} \Lambda_{i} P_{V_{i}} C_{1}^{*} T f \otimes U_{1}^{*} C_{2} P_{W_{j}} \Gamma_{j}^{*} \Gamma_{j} P_{W_{j}} C_{2}^{*} T_{1} g\right) \\
& =\left(\sum_{i \in I} v_{i}^{2} C_{1} U^{*} P_{V_{i}} \Lambda_{i}^{*} \Lambda_{i} P_{V_{i}} T C_{1}^{*} f\right) \otimes \sum_{j \in J} w_{j}^{2} C_{2} U_{1}^{*} P_{W_{j}} \Gamma_{j}^{*} \Gamma_{j} P_{W_{j}} T_{1} C_{2}^{*} g \\
& =C_{1} S_{T U} C_{1}^{*} f \otimes C_{2} S_{T_{1} U_{1}} C_{2}^{*} g \\
& =\left(C_{1} \otimes C_{2}\right)\left(S_{T U} \otimes S_{T_{1} U_{1}}\right)\left(C_{1} \otimes C_{2}\right)^{*}(f \otimes g) \\
& =\left(C_{1} \otimes C_{2}\right) S_{\Delta}\left(C_{1} \otimes C_{2}\right)^{*}(f \otimes g) .
\end{aligned}
$$

Thus, the corresponding frame operator for $\Theta$ is $\left(C_{1} \otimes C_{2}\right) S_{\Delta}\left(C_{1} \otimes C_{2}\right)^{*}$.

In the following theorem, we show that controlled $g$-fusion Bessel sequences in $H$ and $K$ become controlled $g$-fusion frames in $H \otimes K$.

Theorem 9 Let $\Lambda_{T U}=\left\{\left(V_{i}, \Lambda_{i}, v_{i}\right)\right\}_{i \in I}, \Lambda_{T U}^{\prime}=\left\{\left(V_{i}^{\prime}, \Lambda_{i}^{\prime}, v_{i}^{\prime}\right)\right\}_{i \in I}$ be $(T, U)$ controlled $g$-fusion Bessel sequences with bounds $B, D$ in $H$ and $\Gamma_{T_{1} U_{1}}=$ $\left\{\left(W_{j}, \Gamma_{j}, w_{j}\right)\right\}_{j \in J}, \Gamma_{T_{1} U_{1}}^{\prime}=\left\{\left(W_{j}^{\prime}, \Gamma_{j}^{\prime}, w_{j}^{\prime}\right)\right\}_{j \in J}$ be $\left(T_{1}, U_{1}\right)$-controlled $g$-fusion Bessel sequences with bounds $E, F$ in $K$, respectively. Suppose $\left(T_{\Lambda}, T_{\Lambda^{\prime}}\right)$ and $\left(T_{\Gamma}, T_{\Gamma^{\prime}}\right)$ are their synthesis operators such that $T_{\Lambda^{\prime}} T_{\Lambda}^{*}=I_{H}$ and $T_{\Gamma^{\prime}} T_{\Gamma}^{*}=I_{K}$. Then

$$
\Delta=\left\{\left(V_{i} \otimes W_{j}, \Lambda_{i} \otimes \Gamma_{j}, v_{i} w_{j}\right)\right\}_{i \in I, j \in J}
$$

and

$$
\Delta^{\prime}=\left\{\left(V_{i}^{\prime} \otimes W_{j}^{\prime}, \Lambda_{i}^{\prime} \otimes \Gamma_{j}^{\prime}, v_{i}^{\prime} w_{j}^{\prime}\right)\right\}_{i \in I, j \in J}
$$

are $\left(T \otimes T_{1}, U \otimes U_{1}\right)$-controlled $g$-fusion frames for $H \otimes K$.

Proof. By Theorem 4, $\Delta$ and $\Delta^{\prime}$ are $\left(T \otimes T_{1}, U \otimes U_{1}\right)$-controlled $g$-fusion Bessel sequences in $H \otimes K$ with bounds $B E$ and $D F$, respectively. For each $f \otimes g \in H \otimes K$, we can write

$$
\begin{aligned}
\| f \otimes & g \|^{4}=|\langle f \otimes g, f \otimes g\rangle|^{2}=\left|\langle f, f\rangle_{1}\right|^{2}\left|\langle g, g\rangle_{2}\right|^{2} \\
& =\left|\left\langle T_{\Lambda}^{*} f, T_{\Lambda^{\prime}}^{*} f\right\rangle_{1}\right|^{2}\left|\left\langle T_{\Gamma}^{*} g, T_{\Gamma^{\prime}}^{*} g\right\rangle_{2}\right|^{2} \\
& \leq\left\|T_{\Lambda}^{*} f\right\|_{1}^{2}\left\|T_{\Lambda^{\prime}}^{*} f\right\|_{1}^{2}\left\|T_{\Gamma}^{*} g\right\|_{2}^{2}\left\|T_{\Gamma^{\prime}}^{*} g\right\|_{2}^{2}
\end{aligned}
$$




$$
\begin{aligned}
= & \sum_{i \in I}\left(v_{i}^{\prime}\right)^{2}\left\langle\Lambda_{i}^{\prime} P_{V_{i}^{\prime}} U f, \Lambda_{i}^{\prime} P_{V_{i}^{\prime}} T f\right\rangle_{1} \times \sum_{j \in J}\left(w_{j}^{\prime}\right)^{2}\left\langle\Gamma_{j}^{\prime} P_{W_{j}^{\prime}} U_{1} g, \Gamma_{j}^{\prime} P_{W_{j}^{\prime}} T_{1} g\right\rangle_{2} \\
& \times \sum_{i \in I} v_{i}^{2}\left\langle\Lambda_{i} P_{V_{i}} U f, \Lambda_{i} P_{V_{i}} T f\right\rangle_{1} \times \sum_{j \in J} w_{j}^{2}\left\langle\Gamma_{j} P_{W_{j}} U_{1} g, \Gamma_{j} P_{W_{j}} T_{1} g\right\rangle_{2} \\
\leq & D F\|f\|_{1}^{2}\|g\|_{2}^{2}\left(\sum_{i \in I} v_{i}^{2}\left\langle\Lambda_{i} P_{V_{i}} U f, \Lambda_{i} P_{V_{i}} T f\right\rangle_{1}\right) \times \\
& \quad\left(\sum_{j \in J} w_{j}^{2}\left\langle\Gamma_{j} P_{W_{j}} U_{1} g, \Gamma_{j} P_{W_{j}} T_{1} g\right\rangle_{2}\right) \\
= & D F\|f \otimes g\|^{2} \times \sum_{i, j} v_{i}^{2} w_{j}^{2}\left\langle\Lambda_{i} P_{V_{i}} U f \otimes \Gamma_{j} P_{W_{j}} U_{1} g, \Lambda_{i} P_{V_{i}} T f \otimes \Gamma_{j} P_{W_{j}} T_{1} g\right\rangle .
\end{aligned}
$$

Thus, for $h=f \otimes g \in H \otimes K$, we can write

$$
\frac{1}{D F}\|h\|^{2} \leq \sum_{i, j} v_{i}^{2} w_{j}^{2}\left\langle\Delta_{i j} P_{V_{i} \otimes W_{j}}\left(U \otimes U_{1}\right) h, \Delta_{i j} P_{V_{i} \otimes W_{j}}\left(T \otimes T_{1}\right) h\right\rangle .
$$

Hence, $\Delta$ is a $\left(T \otimes T_{1}, U \otimes U_{1}\right)$-controlled $g$-fusion frame for $H \otimes K$. Similarly, it can be shown that $\Delta^{\prime}$ is also a $\left(T \otimes T_{1}, U \otimes U_{1}\right)$-controlled $g$-fusion frame for $H \otimes K$.

Now, we present the frame operator for a pair of $g$-fusion Bessel sequences in $H \otimes K$.

Let $\Lambda_{T U}=\left\{\left(V_{i}, \Lambda_{i}, v_{i}\right)\right\}_{i \in I}$ and $\Lambda_{T U}^{\prime}=\left\{\left(V_{i}^{\prime}, \Lambda_{i}^{\prime}, v_{i}^{\prime}\right)\right\}_{i \in I}$ be $(T, U)$-controlled $g$-fusion Bessel sequences in $H$. The operator $S_{\Lambda \Lambda^{\prime}}: H \rightarrow H$ defined by

$$
S_{\Lambda \Lambda^{\prime}}(f)=\sum_{j \in J} v_{j} w_{j} U^{*} P_{W_{j}} \Lambda_{j}^{*} \Gamma_{j} P_{V_{j}} T f, \quad f \in H,
$$

is called the frame operator for the pair of controlled $g$-fusion Bessel sequences $\Lambda_{T U}$ and $\Lambda_{T U}^{\prime}$.

Let the families

$$
\Delta=\left\{\left(V_{i} \otimes W_{j}, \Lambda_{i} \otimes \Gamma_{j}, v_{i} w_{j}\right)\right\}_{i \in I, j \in J}
$$

and

$$
\Delta^{\prime}=\left\{\left(V_{i}^{\prime} \otimes W_{j}^{\prime}, \Lambda_{i}^{\prime} \otimes \Gamma_{j}^{\prime}, v_{i}^{\prime} w_{j}^{\prime}\right)\right\}_{i \in I, j \in J}
$$

be two $\left(T \otimes T_{1}, U \otimes U_{1}\right)$-controlled $g$-fusion Bessel sequences in $H \otimes K$. The operator $S: H \otimes K \rightarrow H \otimes K$ defined by

$$
S h=\sum_{i, j} v_{i} w_{j} v_{i}^{\prime} w_{j}^{\prime}\left(U \otimes U_{1}\right)^{*} P_{V_{i} \otimes W_{j}}\left(\Lambda_{i} \otimes \Gamma_{j}\right)^{*}\left(\Lambda_{i}^{\prime} \otimes \Gamma_{j}^{\prime}\right) P_{V_{i}^{\prime} \otimes W_{j}^{\prime}} h,
$$

where $h=\left(T \otimes T_{1}\right)(f \otimes g), f \otimes g \in H \otimes K$, is called the frame operator for the pair of $\left(T \otimes T_{1}, U \otimes U_{1}\right)$-controlled $g$-fusion Bessel sequences $\Delta$ and $\Delta^{\prime}$. 
If we put $T=U=I_{H}$ and $T_{1}=U_{1}=I_{K}$ in the above definition, the operator $S$ becomes the frame operator for the pair of $g$-fusion Bessel sequences in $H \otimes K$. Thus, this concept is a generalization of the concept given in [10].

Now, let $\Delta$ and $\Delta^{\prime}$ be two $\left(T \otimes T_{1}, U \otimes U_{1}\right)$-controlled $g$-fusion frames for $H \otimes K$. If we take $v_{i}=v_{i}^{\prime}, V_{i}=V_{i}^{\prime}, \Lambda_{i}=\Lambda_{i}^{\prime}$ and $w_{j}=w_{j}^{\prime}, W_{j}=W_{j}^{\prime}, \Gamma_{j}=\Gamma_{j}^{\prime}$ for each $i \in I$ and $j \in J$, we obtain $\Delta=\Delta^{\prime}$. According to Remark 1, it follows that $S=S_{\Delta}$.

In the next theorem, we establish a relationship between the frame operator for a pair of controlled $g$-fusion Bessel sequences in $H \otimes K$ with those of $H$ and $K$.

Theorem 10 Let $S_{\Lambda \Lambda^{\prime}}$ be the frame operator for the pair of $(T, U)$-controlled $g$-fusion Bessel sequences $\Lambda_{T U}=\left\{\left(V_{i}, \Lambda_{i}, v_{i}\right)\right\}_{i \in I}$ and $\Lambda_{T U}^{\prime}=\left\{\left(V_{i}^{\prime}, \Lambda_{i}^{\prime}, v_{i}^{\prime}\right)\right\}_{i \in I}$ in $H$, and let $S_{\Gamma \Gamma^{\prime}}$ be the frame operator for the pair of $\left(T_{1}, U_{1}\right)$-controlled $g$-fusion Bessel sequences

$$
\Gamma_{T_{1} U_{1}}=\left\{\left(W_{j}, \Gamma_{j}, w_{j}\right)\right\}_{j \in J} \quad \text { and } \quad \Gamma_{T_{1} U_{1}}^{\prime}=\left\{\left(W_{j}^{\prime}, \Gamma_{j}^{\prime}, w_{j}^{\prime}\right)\right\}_{j \in J}
$$

in $K$. Then $S=S_{\Lambda \Lambda^{\prime}} \otimes S_{\Gamma \Gamma^{\prime}}$.

Proof. Noting that $S$ is the associated frame operator for the pair of $\left(T \otimes T_{1}, U \otimes U_{1}\right)$-controlled $g$-fusion Bessel sequences $\Delta$ and $\Delta^{\prime}$, for all $h=$ $f \otimes g \in H \otimes K$, we can write

$$
\begin{aligned}
S h & =\sum_{i, j} v_{i} w_{j} v_{i}^{\prime} w_{j}^{\prime}\left(U \otimes U_{1}\right)^{*} P_{V_{i} \otimes W_{j}}\left(\Lambda_{i} \otimes \Gamma_{j}\right)^{*}\left(\Lambda_{i}^{\prime} \otimes \Gamma_{j}^{\prime}\right) P_{V_{i}^{\prime} \otimes W_{j}^{\prime}}\left(T \otimes T_{1}\right) h \\
& =\sum_{i, j} v_{i} w_{j} v_{i}^{\prime} w_{j}^{\prime}\left(U^{*} P_{V_{i}} \Lambda_{i}^{*} \Lambda_{i}^{\prime} P_{V_{i}^{\prime}} T f \otimes U_{1}^{*} P_{W_{j}} \Gamma_{j}^{*} \Gamma_{j}^{\prime} P_{W_{j}^{\prime}} T_{1} g\right) \\
& =\left(\sum_{i \in I} v_{i} v_{i}^{\prime} U^{*} P_{V_{i}} \Lambda_{i}^{*} \Lambda_{i}^{\prime} P_{V_{i}^{\prime}} T f\right) \otimes\left(\sum_{j \in J} w_{j} w_{j}^{\prime} U_{1}^{*} P_{W_{j}} \Gamma_{j}^{*} \Gamma_{j}^{\prime} P_{W_{j}^{\prime}} T_{1} g\right) \\
& =S_{\Lambda \Lambda^{\prime}}(f) \otimes S_{\Gamma \Gamma^{\prime}}(g)=\left(S_{\Lambda \Lambda^{\prime}} \otimes S_{\Gamma \Gamma^{\prime}}\right)(f \otimes g) .
\end{aligned}
$$

This shows that $S=S_{\Lambda \Lambda^{\prime}} \otimes S_{\Gamma \Gamma^{\prime}}$.

\section{References}

[1] P. Casazza, and G. Kutyniok, Frames of subspaces, Contemporary Math, AMS 345 (2004), pp. 87-114.

[2] O. Christensen, An introduction to frames and Riesz bases, Birkhauser, 2008. 
[3] I. Daubechies, A. Grossmann, and Y. Mayer, Painless nonorthogonal expansions, J. Math. Phys., 27 (1986), no. 5, pp. 1271-1283. https://doi.org/10.1063/1.527388

[4] R.J. Duffin and A.C. Schaeffer, A class of nonharmonic Fourier series, Trans. Amer. Math. Soc., 72 (1952), pp. 341-366. https://doi.org/10.1090/s0002-9947-1952-0047179-6

[5] G.B. Folland, A Course in abstract harmonic analysis, CRC Press BOCA Raton, Florida, 1995.

[6] P. Gavruta, On the duality of fusion frames, J. Math. Anal. Appl., 333 (2007), pp. 871-879.

[7] P. Ghosh and T.K. Samanta, Stability of dual $g$-fusion frame in Hilbert spaces, Methods Funct. Anal. Topol., 26 (2020), no. 3, pp. 227-240. https://doi.org/10.31392/mfat-npu26_3.2020.04

[8] P. Ghosh and T.K. Samanta, Generalized atomic subspaces for operators in Hilbert spaces, Mathematica Bohemica (2021), 21 pages. https://doi.org/10.21136/mb.2021.0130-20

[9] P. Ghosh and T.K. Samanta, Fusion frame and its alternative dual in tensor product of Hilbert spaces, 2021 Preprint, arXiv: 2105.03094, 15 pages.

[10] P. Ghosh and T.K. Samanta, Generalized fusion frame in tensor product of Hilbert spaces, J. Indian Math. Soc. (accepted).

[11] R.V. Kadison and J.R. Ringrose, Fundamentals of the theory of operator algebras, Vol. I, Academic Press, New York, 1983.

[12] A. Khosravi and M.S. Asgari, Frames and bases in the tensor product of Hilbert spaces, Intern. Math. Journal, 4 (2003), no. 6, pp. 527-537.

[13] A. Khosravi and M. Mirzaee Azandaryani, Fusion frames and $g$-frames in tensor product and direct sum of Hilbert spaces, Appl. Anal. Discrete Math., 6 (2012), no. 2, pp. 287-303. https://doi.org/10.2298/aadm120619014k

[14] H. Liu, Y. Huang and F. Zhu, Controlled $g$-fusion frame in Hilbert space, Int. J. Wavelets, Multiresolution Info. Proc., 19 (2021), no. 5, article number 2150009. https://doi.org/10.1142/S0219691321500090

[15] G. Rahimlou, V. Sadri, and R. Ahmadi, Construction of controlled K$g$-fusion frame in Hilbert spaces, U. P. B. Sci. Bull., Series A, 82 (2020), pp. 111-120. 
[16] S. Robinson, Hilbert space and tensor products, Lecture notes, 1997.

[17] V. Sadri, Gh. Rahimlou, R. Ahmadi, and R. Zarghami Farfar, Generalized fusion frames in Hilbert spaces, Infin. Dimens. Anal. Quantum Probab., 23 (2020), no. 2, article number 2050015. https://doi.org/10.1142/S0219025720500150

[18] W. Sun, G-frames and G-Riesz bases, J. Math. Anal. and Appl., 322 (2006), no. 1, pp. 437-452.

[19] G. Upender Reddy, N. Gopal Reddy, and B. Krishna Reddy, Frame operator and Hilbert-Schmidt operator in tensor product of Hilbert spaces, J. Dyn. Syst. and Geom. Theor., 7 (2009), no. 1, pp. 61-70. https://doi.org/10.1080/1726037x.2009.10698563

Prasenjit Ghosh

Department of Pure Mathematics,

University of Calcutta,

35, Ballygunge Circular Road, Kolkata, 700019, West Bengal, India.

prasenjitpuremath@gmail.com

Tapas Kumar Samanta

Department of Mathematics,

Uluberia College, Uluberia,

Howrah, 711315, West Bengal, India.

mumpu_tapas5@yahoo.co.in

Please, cite to this paper as published in

Armen. J. Math., V. 13, N. 13(2021), pp. 1 18

https://doi.org/10.52737/18291163-2021.13.13-1-18 